\title{
Distributed Channel Assignment Algorithm based on Simulated Annealing for Uncoordinated OSA- Enabled WLANs
}

\author{
Francisco Novillo ${ }^{1,2}$, Ramon Ferrús ${ }^{1}$ \\ ${ }^{1}$ Signal Theory and Communications Department, Universitat Politècnica de Catalunya (UPC) \\ Jordi Girona 1-3, Barcelona, Spain \\ \{fnovillo, ferrus\}@tsc.upc.edu \\ ${ }^{2}$ Electrical and Computer Engineering Department, Escuela Superior Politécnica del Litoral (ESPOL) \\ Km 30.5 vía Perimetral, Guayaquil, Ecuador \\ fnovillo@espol.edu.ec
}

\begin{abstract}
A promising approach to alleviate ISM band congestion problems in highly dense WLAN scenarios consists of exploiting opportunistic spectrum access (OSA) to underutilized bands under a primary-secondary model. This paper develops a distributed channel assignment algorithm valid for uncoordinated WLAN deployments where access points do not follow any specific planning and they could belong to different administrative domains. Unlike existing channel assignment schemes proposed for legacy WLANs, channel assignment mechanisms for OSA-enabled WLAN should address two distinguishing issues: channel prioritization and spectrum heterogeneity. Over such a basis, this paper develops and assesses the performance of a distributed channel assignment algorithm that is able to exploit both channel prioritization and spectrum heterogeneity concepts. In particular, the algorithm is based on a distributed adaptation of the simulated annealing metaheuristic algorithm commonly used in global optimization problems.
\end{abstract}

Keywords- Channel Assignment; Distributed Algorithm; OSA; Simulated Annealing; Spectrum Heterogeneity; WLAN.

\section{INTRODUCTION}

Dense deployments of Wireless Local Area Networks (WLANs) are rapidly increasing in urban zones, especially for providing Internet access within residential and office buildings by installing uncoordinated individual access points (AP). These deployments are leading to uncontrolled and excessive levels of interference in unlicensed bands that, ultimately, may turn into both an unpredictable degradation in network performance and unfairness among APs. In these scenarios, distributed channel assignment mechanisms constitute the main tool for reducing as much as possible the level of interference between neighboring WLANs in order not to impair individual network performance. Thus far, WLAN distributed channel assignment problem in ISM bands has received a lot of attention in the research community [1], [2]. However, regardless of the ability of the different channel assignment algorithms to improve WLAN performance, the amount of available spectrum in ISM bands for WLAN use can still constitute a key limiting factor in dense deployments. Hence, the exploitation of additional bands for WLANs (e.g., licensed bands used opportunistically) can help improve the performance of such networks. Potential availability of unused portions of the radio spectrum (i.e., white spaces, WS) to be exploited opportunistically is supported by some recent studies [3]. As a result, major efforts are being conducted in both regulatory and standardization bodies to set out the regulatory and technical framework that will enable opportunistic spectrum access to WSs [4]. Hereafter, WLAN equipment able to use WSs in an opportunistic manner will be referred to as OSA-enabled WLAN.

An uncoordinated deployment of OSA-enabled WLAN may also benefit from having appropriate channel assignment mechanisms to choose the operational channel in each AP among those available either in unlicensed ISM bands or in a given primary band opportunistically exploited. Unlike existing channel assignment schemes proposed for legacy WLANs, channel assignment mechanisms for OSA-enabled WLAN should address two distinguishing issues: (1) channel prioritization, i.e. prioritization criteria other than interference conditions should be considered when choosing between an ISM or a primary channel; and (2) spectrum heterogeneity, i.e. channel availability might not be the same in each AP since it depends on the location and activity of the primary users (PUs). Over such a basis, this paper proposes a distributed channel assignment mechanism that each AP would run asynchronously and that would not require any information exchange between APs (i.e., no coordination between APs). The proposed algorithm is designed to exploit both channel prioritization and spectrum heterogeneity. The proposed mechanism is aimed at keeping interference levels between APs below a certain interference threshold and, at the same time, keeping the utilization of the primary band as low as possible. The latter goal seeks to shield as much as possible the resulting channel allocation from the temporal and spatial variations of the primary channels' availability. The algorithm is built upon a distributed adaptation of the simulated annealing (SA) metaheuristic algorithm widely used in many global optimization problems. Results are provided to assess the benefits of such a proposal under different WLAN deployment densities and primary spectrum availability conditions. As 
well, the algorithm is benchmarked against two well known reference WLAN channel assignment algorithms.

The remainder of the paper is organized as follows. Section II describes the system model characterization. Then, in section III, the theoretical formulation is described and the proposed algorithm is detailed. The performance evaluation of the proposed algorithm is provided in section IV and, finally, concluding remarks and future work are discussed in section V.

\section{SYSTEM MODEL}

\section{A. Network Scenario}

The considered network scenario consists of a set of individual APs (with their associated WLAN client stations) deployed in a limited geographical area. Each AP is expected to operate on an ISM channel or a channel available for opportunistic access in a licensed (primary) band. Licensee users of the primary band are referred to as primary users (PUs) while APs are secondary users (SUs) that can only use that band whenever the operation of PUs is not impaired. Note that channelization used by WLAN in the the primary band (PB) could be different from that used by PUs. Fig. 1 illustrates the envisioned scenario where a dense deployment of OSA-enabled APs coexists with a primary system in the same geographical area.

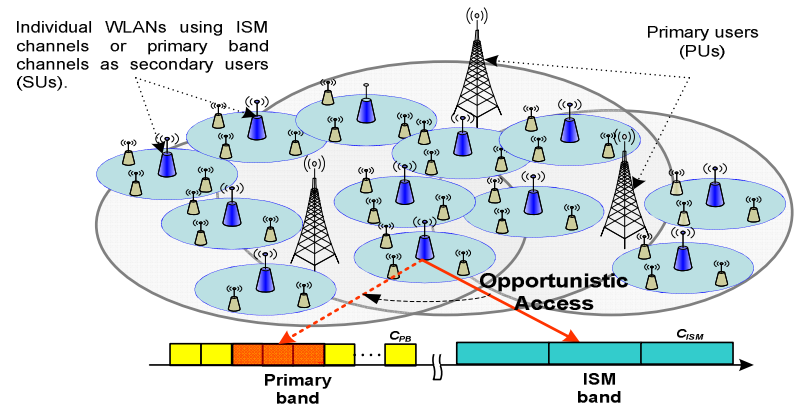

Figure 1. Network scenario

\section{B. Primary Band Availability Conditions}

Availability of primary channels to SUs (i.e. APs) is modeled according to the locations and the potential interference between PUs and SUs. In particular, conditions for determining which primary channels can be used by SUs are formulated in terms of the maximum interference levels that can be tolerated by both the PU and SU receivers. Hence, a SU can use a given primary channel whenever the interference received by any PU receiver tuned at that channel, $I_{S P}$, is below the PU receiver sensitivity $S_{P}$ minus a given protection margin $M_{P}$. This usage condition imposed on SU transmitters can be formulated as $I_{S P} \leq S_{P}-M_{P}$. Additionally, the successful operation of SU receivers tuned into primary channels also requires interference received from PU transmitters, $I_{P S}$, to be lower than SU receiver sensitivity minus a protection margin $M_{S}$. Hence, the usage condition required by $\mathrm{SU}$ receivers can be formulated as $I_{P S} \leq S_{S}-M_{S}$. It is worth noting that both receiver protection margins, $M_{S}$ and $M_{P}$, would account for the fading margin along with the minimum required signal-tointerference ratio. The two usage conditions can be used to
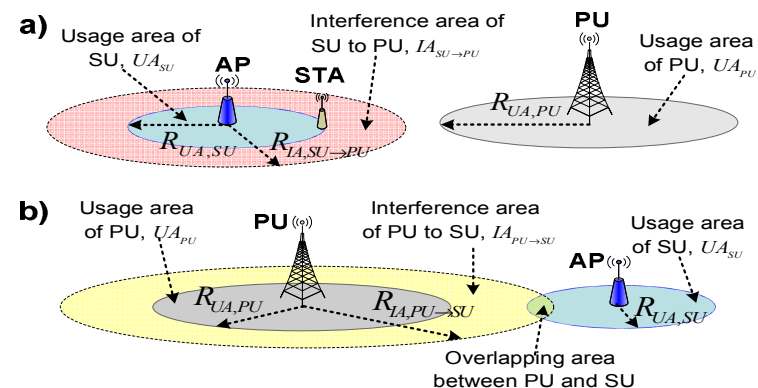

Figure 2. Interference conditions: a) From WLAN to PUs, b) From PU to WLANs.

define a set of usage and interference areas for PUs and SUs. The usage area is the coverage area of a given service. Interference area represents the spatial area in which a receiver would be exposed to an unacceptable level of interference generated by a transmitter. Hence, considering omnidirectional antennas and homogeneous propagation conditions, spatially, these areas would be circular in shape, as illustrated in Fig. 2. Assuming a propagation model characterized by channel attenuation at $1 \mathrm{~m}\left(L_{o}\right)$ and propagation slope $\alpha$, the radius for the usage area $\left(R_{U A, x}\right)$ is computed by means of the following expression: $R_{U A, x}=10^{\frac{\left(P_{x}-S_{x}\right)-L o}{10 \alpha}}$, in which $x$ can be either PU or $\mathrm{SU}$, and the pair $\left(P_{x}, S_{x}\right)$ is the transmitted power and sensitivity of the considered system. Building upon the concepts of usage and interference areas, an Interference Penalty $(I P)$ factor is defined as the percentage of the usage area in which the correspondent availability condition will not be met. The IP factor is computed as:

$$
I P\left(y^{i}, z^{j}\right)=\frac{\left[U A_{z} \cap I A_{y \rightarrow z}\left(\rho_{y^{i} \rightarrow z^{j}}\right)\right]}{U A_{z}}
$$

where $y$ and $z$ represent the interfering and interfered device respectively, which can be either PU or SU. $U A$ is the usage area of $z$ and $I A$ is the interference area between $y$ and $z . i, j$ correspond to transmitter and receiver channels, respectively. $\rho_{y^{i} \rightarrow z^{j}}$ is the overlapping interference factor, which corresponds to the normalized received power at the output of the receiver filter defined in the model of partially-overlapping wireless channels developed in [5]. Relying on the IP factor definition, the possibility of using a given primary channel in an SU is determined according to the accomplishment of the following two conditions: a) The usage area of a PU must not overlap with the interference area of a SU (i.e., $\left.I P\left(S U^{i}, P U^{j}\right)=0\right)$. Thus, since PUs have a priority use on the primary band, the SUs are not allowed to cause interference within the coverage range of the PUs, as shown in Fig. 2(a). b) The amount of overlapping between the usage area of a SU and the interference area of a PU must not exceed a certain threshold $\left(I P_{M A X}\right)$, (i.e. $\left.I P\left(P U^{i}, S U^{j}\right) \leq I P_{M A X}\right)$.

\section{Channel Assignment Constraints}

The IP factor is also used to establish channel allocation constraints for the individual WLAN networks. In particular, a given pair of APs $\left(a p_{u}\right.$ and $\left.a p_{v}\right)$ is allowed to use a given pair of 
channels ( $i$ and $j$ ) when the following condition is satisfied: $I P\left(a p_{u}^{i}, a p_{v}^{j}\right) \leq I P_{M A X}$. The same condition applies regardless of whether the channels being considered are ISM or PB channels.

\section{Distributed CHANNEl ASSIGNMENT AlgORITHM}

In this section, a distributed channel assignment mechanism that exploits both channel prioritization and spectrum heterogeneity is developed by means of a heuristic algorithm based on simulated annealing techniques. The algorithm is executed by each AP in a distributed manner and is only based on local information (i.e., no information exchange takes place between neighboring APs). The objective of the algorithm is to find a proper channel assignment for every AP so that the IP factor between any pair of APs $\operatorname{IP}\left(a p_{u}^{i}, a p_{v}^{j}\right)$ is kept below a certain threshold $I P_{M A X}$ and the number of APs using PB channels is minimized. The rationale behind pursuing a low usage of PB channels is that we are interested in finding a solution with low dependability on the presence of primary users.

\section{A. Problem Formulation}

To formulate this problem in a mathematical way, in the following, $V=\left\{a p_{1}, a p_{2}, \ldots, a p_{n_{S}}\right\}$ corresponds to the $n_{s}$ APs in the network scenario. The set of available channels for $a p_{u}$ is defined as: $A\left(a p_{u}\right)=\left\{a_{i} \mid a_{i} \in\{0,1\} \forall 1 \leq i \leq C_{T}\right\}$, where $a_{i}=1$, if channel $i$ is available at $a p_{u}, C_{T}=C_{I S M}+C_{P B}$, and $C_{I S M}$ and $C_{P B}$ are the number of available channels for WLAN operation in the ISM and PB bands, respectively. The channel assigned to $a p_{u}$ is denoted as: $C\left(a p_{u}\right)=\left\{i \mid 1 \leq i \leq C_{T} \wedge a_{i}=1\right\}$. Two APs are considered to be neighbors if the $I P$ factor calculated under co-channel conditions is greater than zero. Thus, the set of neighbors for $a p_{u}$ is defined as: $N\left(a p_{u}\right)=\left\{a p_{v} \mid \forall a p_{v} \in V, I P\left(a p_{u}^{i}, a p_{v}^{i}\right)>0\right\}$. The maximum interference resulting at $a p_{u}$ from its neighbors when channel $i$ is used is computed by means of the following expression: $M I P_{a p_{u}}^{i}=\max _{\forall a p_{v} \in N\left(a p_{u}\right)} I P\left(a p_{u}^{i}, a p_{v}^{C\left(a p_{v}\right)}\right)$, If $M I P_{a p_{u}}^{i}$ is below the threshold $I P_{M A X}$, then the channel $i$ is considered as a feasible channel for the $a p_{u}$. In accordance with the above definitions a utility function $U\left(M I P_{a p_{u}}^{i}\right)$ is used to map $M I P_{a p_{u}}^{i}$ values to the preference given to channel $i$ by $a p_{u}$ when looking for an operational channel. The utility function is a decreasing function with respect to the amount of MIP so that the lower the $M I P$ for a given channel, the higher the utility given to that the channel. In particular, a sigmoid function defined as follows is used in our analysis [6]:

$$
U\left(M I P_{a p_{u}}^{i}\right)= \begin{cases}1-(1-q) \cdot e^{s\left(M I P_{a p_{u}}^{i}-I P_{M A X}\right)} & ; M I P_{a p_{u}}^{i} \leq I P_{M A X} \\ q \cdot e^{-s\left(M I I_{a p_{u}}^{i}-I P_{M A X}\right)} & ; \text { otherwise }\end{cases}
$$

where $q$ denotes the utility value when $M I P_{a p_{u}}^{i}=I P_{M A X}$, and $s$ determines the slope of the utility function. The objective of the channel assignment problem is then set out to maximize the utility function. Hence, the channel assignment problem for each AP can be simply formulated as:

$$
\operatorname{maximize}\left[U\left(M I P_{a p_{u}}^{i}\right)\right]
$$

Subject to: $a p_{u}$ only uses one channel at a time

\section{B. Algorithm Description}

The algorithm is executed locally at each AP periodically and allows each one to select its operation channel, either from an ISM band or a primary band. The algorithm is made using the simulated annealing technique that uses a stochastic approach to direct the search of a channel assignment and targeted to maximize the utility function of the AP. The local search method is shown in Fig. 3. Notation of Fig. 3 for the utility function has been simplified because, as the algorithm is executed at each AP, the utility of that particular AP is just expressed as $U\left(M I P_{a p_{u}}^{i}\right)=u(i)$. The algorithm works as follows: At each access point, the algorithm starts if any change in the network environment of the AP is detected (e.g., number of neighbors, number of available primary channels, etc). In the first case, the algorithm computes an initial channel, $i$ that is assigned to the AP as the current operation channel; also, SA parameters are set up i.e., initial temperature, $T_{0}$, cooling rate $C R$, and the predefined constant $\in$ (line: 1 ). The algorithm is executed iteratively. Thereby, a new channel, $j$ is obtained as a candidate channel to be assigned to the AP at each iteration (line: 3). The way the candidate channel is chosen is described below. The decision to hold the current channel, $i$ or assign the candidate channel, $j$ depends on a comparison between utilities obtained by those channels. Therefore, if $u(i)$ is the utility obtained with the current channel and $u(j)$ with the candidate channel then the candidate channel is assigned to the AP if the utility is incremented i.e. $\delta=u(i)$ $u(j)<0$ (line: 5-6). If the candidate channel decreases the utility, it will be accepted with probability depending on the change in utility $\delta$ and the current temperature $T, \operatorname{Pr}[\delta, T]=\mathrm{e}^{-\delta / T}$ (line: 7-9). On the other hand, if the candidate channel provides the same utility as the current channel, it will be accepted with probability depending on the predefined constant, $\in$ and $T$, $\operatorname{Pr}[\epsilon, T]=\mathrm{e}^{-\epsilon / T}$ (line: 11-13). Otherwise, the operation channel does not change. It is this stochastic selection scheme that helps SA to avoid being stuck at local optimum. $T$ values are updated at each iteration (line: 16) and gradually decrease so that the probability of accepting a new channel that does not improve the utility also decreases. To obtain a candidate channel, the algorithm assigns a probability to each channel at the AP as shown in the following expression: $p_{i}=\frac{w_{i}}{\sum w_{i}}, \forall i$, being $w_{i}$ the weight of each channel, so that, if there is at least one feasible channel then $w_{i}=u_{i}$ for the feasible channels, and $w_{i}=0$ otherwise. Notice that, if $M I P_{a p_{u}}^{i} \leq I P_{M A X}$ then the channel $i$ is considered to be a feasible channel. If the channels are not feasible, $w_{i}=u_{i}$. Additionally, in the case that there is at least one feasible channel in each band, weights of the primary channels are multiplied by the band prioritization factor $B P$ i.e., 
$w_{i}=B P . u_{i}$, where $B P=[0,1]$. Notice that, when channels are not feasible, the priority for both bands is the same, so that the channels with more utility have a higher probability of being chosen.

\section{PERFORMANCE EVALUATION}

In this section, a performance analysis of the proposed algorithm is carried out under different conditions of APs' density and primary spectrum heterogeneity. The performance of the proposed distributed algorithm (DA) is also compared to two other channel assignment algorithms [1]: LCCS (Least Congested Channel Search), where each AP chooses the channel with the least number of other APs assigned among its neighbors, and ADJ-minmax, where an AP computes for each available channel, the maximum interference that would have with its neighbors and chooses the channel with the minimum value of maximum interference. In the following ADJ-minmax is referred to as MM. Additionally, for all evaluations, initial channel assignment is obtained by implementing the FF algorithm proposed in [7]. To that end, topology snapshots with random spatial distributions of primary and secondary users on a normalized area of $1 \times 1$ are generated to solve the channel allocation problem. In this particular study, for the sake of simplicity, the spectrum transmission masks for primary and WLAN users are considered to have rectangular forms, as shown in Fig. 1. Each primary user operates a given channel of the primary band (randomly selected in this study). Primary band has ten non-overlapping $5 \mathrm{MHz}$ bandwidth channels. Hence, depending on the location of primary users and APs, and on the channels used by the primary users, the APs can have between 0 and 10 additional (overlapped) channels in the primary band, in addition to $C_{I S M}=11$ overlapped channels in the ISM band. Provided results have been obtained from 5000 snapshots for $I P_{M A X}=0.2, M_{S}=10 \mathrm{~dB}$, $M_{P}=15 \mathrm{~dB}, S_{S}=-65 \mathrm{dBm}, S_{P}=-65 \mathrm{dBm}, \alpha=3.5$, Radio operation parameters of the two systems have been chosen to have the following usage and interference area radii: $R_{U A, S U}=0.05$, $R_{U A, P U}=0.051, \quad R_{I A, P U \rightarrow S U}=0.1, \quad R_{I A, S U \rightarrow P U}=0.18 \quad$ and $R_{I A, S U \rightarrow S U}=0.18$ Moreover, SA parameters $T_{0}=85, \epsilon=5$ are established in order to obtain a higher probability for accepting a channel with a utility less than or equal to the utility of the current operation channel in the initial iterations.

Considering an initial scenario with densities of 72 APs and 20 PUs, Fig. 4 shows the percentage of APs that are able to find a feasible channel (in the following, an AP with a feasible channel is going to be referred as a feasible AP) together with the average number of iterations that each AP needs to run before converging, both for different values of utility slope $(s)$ and cooling rate $C R$. As shown in graph a) of Fig 4, $s$ ' values around 10 are found to have the highest values of feasibility. This is because, for this range, the values of channel utility obtained by the sigmoid function (2) are farther from each other and, as a result, the probabilities assigned to each channel are as well. Hence, for this $s$ ' range, the probability of choosing a channel with a high utility is higher than for other $s$ 'values. In addition, this fact allows the algorithm to require less iterations to converge, as shown in Fig. 4 b). This is due to the fact that the probability of changing to a channel with less

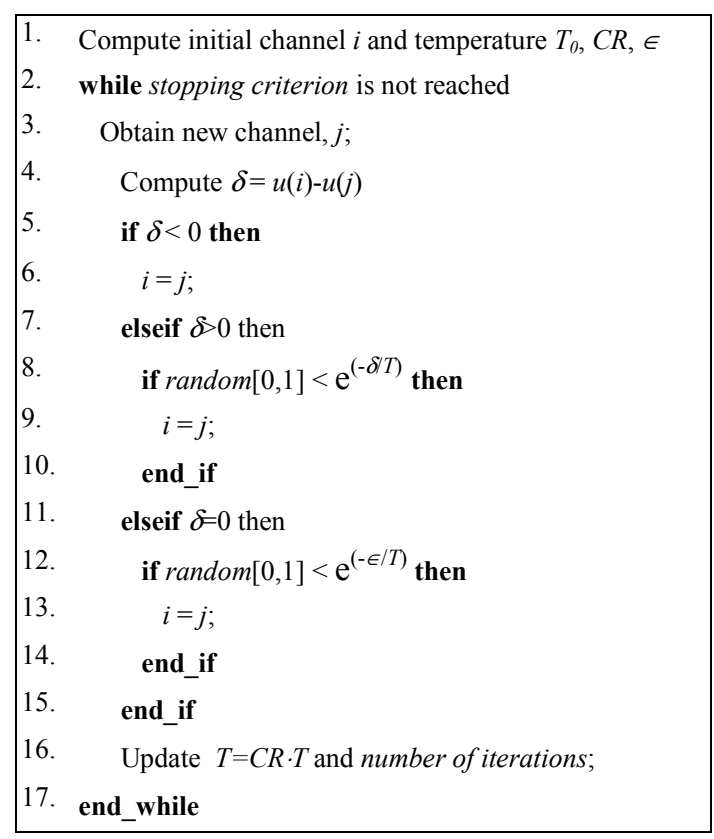

Figure 3. Pseudocode of the distributed SA algorithm

utility than the current channel is decreased, since $|\delta|$ in line 8 from Fig. 3 is increased. Also, it is observed that, the algorithm obtains more feasible APs when $C R$ is increased. This increase of $C R$ causes the temperature $(T)$ to decrease more slowly; consequently, the probabilities (i.e., line: 8, 12 from Fig. 3) of the SA algorithm decrease, too, so that the algorithm could perform more iterations to find better channels and not stay with a local optimum solution. For instance, for $s=10$, DA with $C R=0.8$ obtains around $4.7 \%$ more feasible APs and requires around 17 more iterations than with $C R=0.1$. Additionally, from Fig.4, the performance of DA is better than that obtained by MM. For instance, for $s=10$, and $C R=0.6$, DA obtains around $6 \%$ more feasible APs and around 4 more iterations than MM. In conclusion, if in each iteration the algorithm chooses the operation channel from a set of feasible channels (i.e., DA) better solutions are obtained than if the algorithm directly chooses the best feasible channel (i.e., MM). In the following, the values used for $s$ and $C R$ are 10 and 0.6 respectively. Those value are chosen since $C R=0.6$ provides a percentage of feasible APs close to that obtained with $C R=0.8$ and requires around 11 less iterations; likewise, for $s=10$ the highest number of feasible APs with the least number of iterations is obtained. After studying parameters of the algorithm, the results obtained are that, for $B P$ values, from 0 up to 1 , the percentage of feasible access points obtained is up to $0.5 \%$ higher than that obtained by $B P=0$. In the same way, the number of APs using primary band increases by up to around $16 \%$. Hence, in order to obtain a good percentage of feasible APs and a reasonable use of primary band, the value chosen for $B P$ is 0 .

Fig. 5 a) provides the percentage of feasible APs for different densities of APs within the scenario. As shown in the figure, the best performance is that obtained by DA, followed by the MM, and finally by the LCCS, for either ISM band or both ISM and primary bands (e.g., for 72 APs considering ISM+PB bands, DA is able to provide $6 \%$ and $30 \%$ more 
feasible APs than MM and LCCS, respectively). Fig. 5 b) shows the percentage of APs using primary band required for obtaining the feasible APs shown in Fig. 5 a). This figure illustrates the percentage of APs using primary band for the DA algorithm is always much lower than the reference techniques (i.e., MM, LCCS). For instance, for 72 APs, DA has 9.3\% and $13.1 \%$ less APs using PB than with MM and LCCS, respectively. In conclusion, the prioritization of the ISM band in the DA algorithm allows the APs to be less dependent on the primary channels, and, consequently, makes for a more efficient use of the ISM band. Fig. 6 depicts the percentage of feasible APs and the percentage of feasible scenarios (i.e., snapshots with all AP with IP below $\left.I P_{M A X}\right)$ that are obtained when the spectrum heterogeneity is increased (i.e., density of PUs is increased). The results obtained by DA are always better than those obtained by the other algorithms. DA is more stable than the other algorithms for changes in the spectrum heterogeneity; this can be observed in the percentage of feasible scenarios obtained by DA that decreases more slowly than other algorithms.

\section{CONCLUSIONS}

This paper has proposed and evaluated the performance of a distributed algorithm designed for opportunistic channel allocation in densely uncoordinated WLAN scenarios. The algorithm supports the prioritization of ISM in front of PB channels and exploits the fact that $\mathrm{PB}$ availability is not homogeneous in order to better assign channels to APs. The algorithm has been shown to considerably increase the probability of finding feasible assignment solutions while achieving a low usage of the primary channels compared to other classical WLAN algorithms such as LCCS and ADJminmax. Provided results also show that the computational load of the proposed algorithm is similar to that of reference schemes.

\section{ACKNOWLEDGMENT}

This work was supported by the Spanish Research Council and FEDER funds under ARCO grant (ref. TEC2010-15198).

\section{REFERENCES}

[1] Mishra, A., Banerjee, S., and Arbaugh, W. 2005. Weighted coloring based channel assignment for WLANs. SIGMOBILE Mob. Comput. Commun. Rev. 9, 3 (Jul. 2005), 19-31.

[2] Xiaonan Yue; Chi-Fai Wong; Chan, S.-H.G.; , "A Distributed Channel Assignment Algorithm for Uncoordinated WLANs," Consumer Communications and Networking Conference (CCNC), 2010 7th IEEE, vol., no., pp.1-5, 9-12 Jan. 2010

[3] Lopez-Benitez, M.; Casadevall, F.; Umbert, A.; Perez-Romero, J.; Hachemani, R.; Palicot, J.; Moy, C., "Spectral occupation measurements and blind standard recognition sensor for cognitive radio networks," Cognitive Radio Oriented Wireless Networks and Communications, 2009. CROWNCOM'09. 4th International Conference on, vol., no., pp.1-9, 22-24 June 2009.

[4] European Telecommunications Standards Institute (ETSI): Technical Report Reconfigurable Radio Systems (RRS); Use cases for Operation in White Space Frequency Bands. Draft ETSI TR 102907 V0.0.8 (2010)

[5] Mishra, A., Shrivastava, V., Banerjee, S., and Arbaugh, W. 2006. Partially overlapped channels not considered harmful. In Proceedings of the Joint international Conference on Measurement and Modeling of
Computer Systems (Saint Malo, France, June 26 - 30, 2006). SIGMETRICS '06/Performance '06. ACM, New York, NY, 63-74.

[6] Wen-Hsing Kuo and Wanjiun Liao, "Utility-based Radio Resource Allocation for QoS Traffic in Wireless Networks" Wireless Communications, IEEE Transactions on, vol. 7, no., pp. 2714 - 2722, July 2008

[7] Novillo, F.; Ferrus, R.; Agusti, R.; Nasreddine, J., " Opportunistic Channel Allocation Algorithms for WLANs Based on IEEE802.11," Future Network Summit 2010 Conference Proceedings. Jun 2010.
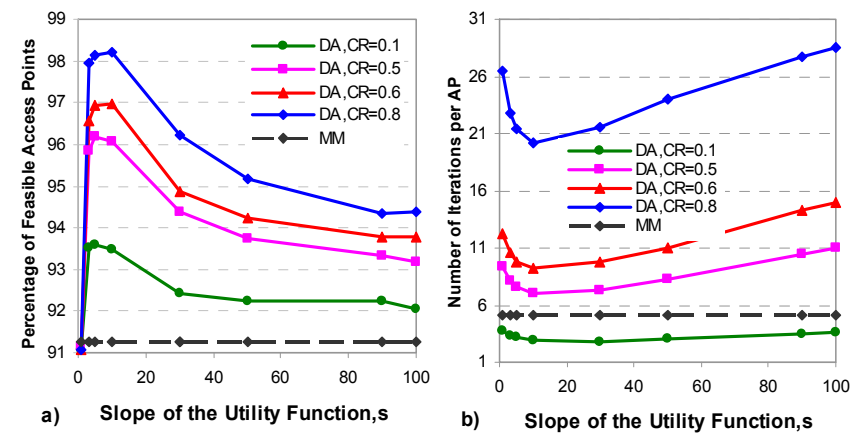

Figure 4. Convergence Analysis: a) Percentage of Feasible Access Points versus Number of Access Points. b) Number of Iterations per Access Point versus Slope of the Uyility Function, for $B P=0$, and densities of 72 APs and 20 PUs.
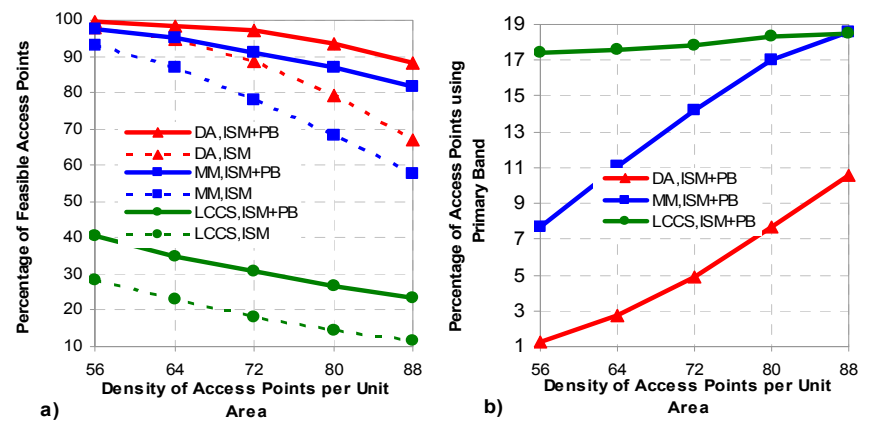

Figure 5. a) Percentage of Feasible Access Points versus Density of Access Points per Unit Area. b) Percentage of Access Points using Primary Band versus Density of Access Points per Unit Area, for a density of 20 PUs.

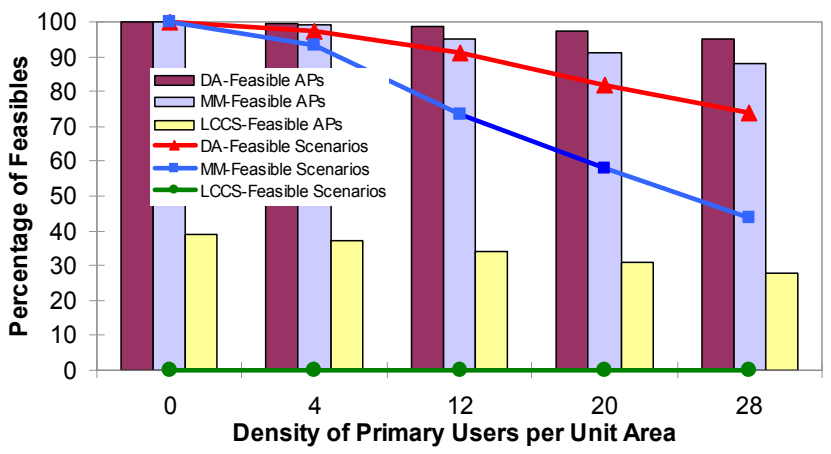

Figure 6. Both Percentage of Feasible Assignments and Percentage of Feasible Access Points versus Density of Primary Users per Unit Area, for a density of 72 APs. 